\section{Attacking the mediator}

Plants use a range of mechanisms to respond to challenge by plant pathogens and, in turn, plant pathogens use a range of mechanisms to interfere with and evade these responses. Plant defence responses include those that are mediated by salicylic acid and jasmonic acid-ethylene but, so far, few phytopathogen effectors that interfere with these hormone-based systems have been identified. A study in PLoS Biology now shows that an effector from the biotrophic oomycete pathogen Hyaloperonospora arabidopsidis attenuates the salicylic acid response, thus enhancing biotrophy.

Many oomycete effectors contain an amino-terminal RXLR (where $\mathrm{X}$ is any amino acid) motif that correlates with entry into host cells. Previous characterization of the RXLR effector repertoire of $H$. arabidopsidis identified a subset of effectors that localize to the plant cell nucleus and interact with components of the Mediator complex - a multisubunit transcriptional regulation complex that is present in all eukaryotes. In plants, Mediator has been shown to influence key processes, including plant development and, recently, plant immunity.

Caillaud and colleagues present an in-depth characterization of one of the $H$. arabidopsidis RXLR nuclear effectors, HaRxL44, which interacts with the MED19a subunit of the Mediator complex. Analysis of transgenic Arabidopsis thaliana lines showed that those in which MED19a was non-functional were more susceptible than the wild type to infection with $H$. arabidopsidsis. By contrast, transgenic lines in which MED19a was overproduced were more resistant to infection than the wild type. This indicates that MED19a is a positive regulator of $A$. thaliana immunity to $H$. arabidopsidsis infection. Confocal microscopy to monitor the subcellular localization proteins showed that both localize to the plant nucleolus and nucleoplasm. However, colocalization analysis showed that when HaRxL44 was present in the nucleoplasm MED19a could not be detected. Further investigations revealed that HaRxL44 degrades MED19a in a proteasome-dependent manner.

To pinpoint the mechanism by which HaRxL44-mediated MED19a degradation affects the immune response to $H$. arabidopsidsis infection, the authors used quantitative reverse transcription-PCR analysis and gene expression profiling. They found that, in the presence of HaRxL44, marker genes that were consistent with jasmonic acidethylene signalling were upregulated, whereas marker genes that were consistent with salicylic acid of MED19a and HaRxL44 fusion

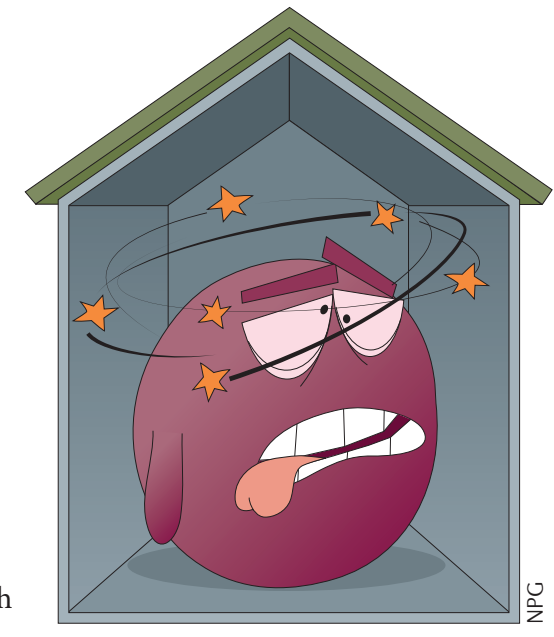

signalling - which has a key role in the response to biotrophic pathogens - were downregulated. Finally, the authors investigated the cell-specific expression patterns of marker genes that are associated with the salicylic acid response and found that H. arabidopsidsis infection only suppresses the salicylic acid response in those cells that have been parasitized by oomycete haustoria.

These data show that the H. arabidopsidsis HaRxL44 effector targets MED19a to modulate the balance between jasmonic acid-ethylene and salicylic acid signalling, such that the salicylic acid response is attenuated and biotrophic infection is favoured. It will be interesting to follow future studies that characterize the functions of the other H. arabidopsidsis RXLR nuclear effectors.

Sheilagh Molloy

ORIGINAL RESEARCH PAPER Caillaud, M.-C. et al. A downy mildew effector attenuates salicylic acid-triggered immunity in Arabidopsis by interacting with the host mediator complex. PLoS Biol. 11, e1001732 (2013) 